\title{
As causas da deficiência física em municípios do nordeste brasileiro e estimativa de custos de serviços especializados
}

\author{
The causes of physical disability in municipalities of the northeast \\ of Brazil and an estimate of costs of specialized services
}

\begin{abstract}
Silvana Santos ${ }^{1}$
Anne Aluska da Silva Pequeno ${ }^{1}$

Cláudia Regina Cabral Galvão ${ }^{2}$

André Luiz Santos Pessoa ${ }^{3}$

Ednno dos Santos Almeida ${ }^{4}$

Josecleide Calixto Pereira ${ }^{4}$

Jovany Luiz Alves de Medeiros ${ }^{1}$

Fernando Kok ${ }^{5}$
\end{abstract}

${ }^{1}$ Programa de Mestrado em Saúde Pública,

Departamento de Biologia, Universidade Estadual da Paraíba. Rod PB 075 2001, Zona Rural. 58.200-000 Campina Grande PB Brasil.silvanasantos@ ccbs.uepb.edu.br

${ }^{2}$ Departamento de Terapia

Ocupacional, Universidade Federal da Paraíba.

${ }^{3}$ Curso de Medicina, Universidade de Fortaleza.

${ }^{4}$ Departamento de Biologia, Universidade Estadual da Paraíba.

${ }^{5}$ Departamento de

Neurologia, Hospital das Clínicas, Universidade de São Paulo.
Abstract The population of the northeast of Brazil is characterized by high rates of endogamy and disabilities. An epidemiological cross-sectional study using the informant method was conducted in eight communities in the hinterlands of Paraiba to describe genetic and acquired diseases that cause disabilities and to estimate the costs of specialized services such as physiotherapy and the acquisition of technological assistential equipment. From a population of 48,499 inhabitants, 338 individuals were screened and 123 (0.34\%) were clinically, genetically and functionally assessed by a multidisciplinary team of specialists. Genetic factors were responsible for $58.5 \%$ of the disabilities, with some clusters of prevalent diseases being found within the sampled communities, namely progressive spinal muscular atrophy, spinocerebellar ataxia, muscular dystrophy and Spoan syndrome. The socioeconomic profile and the demand for rehabilitation services and technological assistance highlight the need to introduce and implement specific public health policies in these communities.

Key words Disability, Epidemiology, Consanguinity, Community Assistential and Genetic Technology
Resumo As populações do nordeste brasileiro possuem elevadas taxas de endogamia e deficiências. Neste trabalho, foi realizado um estudo epidemiológico transversal com método do informante para descrever doenças genéticas e as adquiridas que causam deficiências físicas em oito municípios da Paraíba e estimar os custos por serviços especializados de fisioterapia e aquisição de equipamentos de tecnologia assistiva. De uта рориlação de 48.499 habitantes, 338 pessoas foram triadas e $123(0,34 \%)$ realizaram avaliação clínicogenética e funcional por uma equipe multidisciplinar de especialistas. Fatores genéticos foram responsáveis por $58,5 \%$ das deficiências, sendo encontrados alguns agrupamentos de afecções prevalentes nas populações amostradas: amiotrofia espinal progressiva, ataxia espinocerebelar, distrofia muscular e síndrome Spoan. A descrição do perfil socioeconômico e das demandas por serviços de reabilitação e tecnologia assistiva apontam para necessidade de estabelecimento de políticas públicas específicas para essas comunidades.

Palavras-chave Deficiência, Epidemiologia, Consanguinidade, Tecnologia assistiva, Genética comunitária 


\section{Introdução}

Há dez anos, as pessoas com deficiências e incapacidades representavam $14,5 \%$ da população brasileira $^{1,2}$ e atingiram a fração de $23,9 \%$ dos 190.732.694 brasileiros no censo demográfico de $2010^{3}$. Em relação à distribuição regional, foi observada a existência de assimetrias regionais e as pessoas com deficiência são encontradas mais frequentemente nos estados do nordeste brasileiro. O Rio Grande do Norte e a Paraíba possuem praticamente $18 \%$ de sua população acometida por alguma deficiência e lideram, há vinte anos, o "ranking da deficiência" no Brasil. Não existem evidências, até o presente momento, de que a pobreza seja a causa determinante desse fenômeno ${ }^{4}$; tendo em vista que existem outros fatores de natureza ambiental, genética ou demográfica, contribuindo para essa situação. Se conhecermos mais profundamente a etiologia e os fatores determinantes das deficiências, será possível planejar políticas públicas, ações mitigatórias e preventivas para essas populações.

Curiosamente, a endogamia ou os casamentos consanguíneos são também bastante frequentes nos estados do nordeste brasileiro. Estudos feitos em meados do século passado mostraram que em média 9\% dos casamentos ocorriam entre casais aparentados $s^{5-8}$ e essas estimativas não se reduziram desde então. Levantamentos recentes feitos em municípios do sertão do Rio Grande do Norte e da Paraíba mostraram que de 9\% a $41 \%$ dos casamentos são consanguíneos ${ }^{9-11}$. Nessa região, foi descoberta uma doença neurodegenerativa, a síndrome Spoan, que afeta mais de 70 pessoas de uma mesma famíliaa ${ }^{12,13}$. Essa doença genética, prevalente em Serrinha dos Pintos e São Miguel (RN), também é um dos principais fatores etiológicos das deficiências físicas na região ${ }^{10}$.

Esses achados indicaram que parte das deficiências encontradas no sertão nordestino é causada por doenças genéticas. Neste trabalho, foi realizado levantamento das deficiências que acarretam dificuldade ou incapacidade de locomoção, ou seja, função motora, em oito municípios da Paraíba com elevadas taxas de casamentos consanguíneos; os quais fazem divisa com a região onde a síndrome Spoan é prevalente. Os escopos desta pesquisa são, por um lado, descrever as doenças que causam deficiências físicas nessa população e, por outro, levantar as demandas por serviços de reabilitação e tecnologia assistiva.

O custo da oferta desses serviços para o Estado foi estimado com a finalidade de determinarmos quanto seria necessário investir para que as pessoas que necessitam de fisioterapia e equipamentos de tecnologia assistiva pudessem ter acesso a esses serviços especializados. Os resultados deste estudo podem contribuir para o planejamento e a execução de políticas públicas para pessoas com deficiência que vivem em regiões do nordeste brasileiro e/ou com características socioculturais semelhantes.

\section{Métodos}

\section{População Amostrada e Procedimentos}

Este é um estudo de base populacional transversal com uso do método do informante. O levantamento das deficiências físicas que comprometem a marcha e a função motora foi realizado em oito municípios do Estado da Paraíba, os quais participaram de um estudo prévio sobre padrões reprodutivos e endogamia ${ }^{11}$. Esses municípios estão localizados em áreas rurais, relativamente isoladas, distando $350-400 \mathrm{~km}$ da capital do estado, João Pessoa; e possuem elevadas frequências de casamentos consanguíneos. A população total envolvida nesta pesquisa, segundo estimativa preliminar do censo demográfico ${ }^{3}$, é de 48.499 habitantes, distribuídos nos seguintes municípios: Bernardino Batista com 3.067 habitantes; Jericó, 7.535; Lagoa, 4.681; Poço Dantas, 3.752; Santa Cruz, 6.471; São Francisco, 3.364; Vieirópolis, 5.045 e Uiraúna com 14.584 habitantes.

Os profissionais que atuam no Programa de Saúde da Família (agentes comunitários de saúde e enfermeiros) participaram de um curso de formação continuada durante o ano de 2010 e colheram informações a respeito da frequência de casamentos consanguíneos em seus municípios; bem como tiveram acesso às informações sobre epidemiologia e genética básica. No período de janeiro a abril de 2010, os agentes comunitários de saúde participaram novamente de uma série de seminários de formação para treinamento a fim de realizarem entrevistas com pessoas com deficiência usando um roteiro estruturado, selecionando assim aquelas pessoas que apresentavam dificuldade ou incapacidade de locomover-se para avaliação clínico-genética e funcional realizada por especialistas. Para evitar qualquer perda e vieses, $10 \%$ dos pacientes indicados pelos agentes de saúde foram novamente contatados por telefone para checar os dados presentes na ficha de triagem. Estudos para checar a estratégia, validação de instrumentos de coleta e vieses já foram realizados anteriormente ${ }^{9,11}$, e neles se 
verificou erros de preenchimento na ficha de triagem da ordem de três por cento. Como essa ficha é relativamente simples, e nela constam apenas informações como nome, endereço, telefone dos entrevistados e descrições, nas palavras dos agentes de saúde, sobre o quadro do paciente e se ele já foi ou não atendido por médicos especialistas, bem como se tem ou não um laudo com diagnóstico clínico; então esse instrumento acaba por desempenhar a função de facilitar a organização da triagem realizada posteriormente. Os agentes de saúde foram instruídos a incluir todas as pessoas com dificuldade de locomoção e cadeirantes, o que é um procedimento relativamente simples. Além disso, as fichas de triagem preenchidas por eles eram encaminhadas aos enfermeiros que também participaram dos seminários de formação, o que reduziu os riscos de perdas ou vieses.

Ao todo, foram preenchidas 338 fichas de triagem, tendo sido registradas 161 pessoas com alguma forma de deficiência física e, deste subconjunto, 123 delas tinham perda de função motora representando $0,34 \%$ da população total dos municípios amostrados. Esses 123 indivíduos realizaram a avaliação clínico-genética com cinco professores doutores e especialistas em Neurologia e Genética Médica para determinação de diagnóstico; e a avaliação físico-funcional foi feita por dois profissionais, especialistas em fisioterapeuta e terapeuta ocupacional, com utilização de um protocolo desenvolvido pelo grupo. Todos os pacientes foram avaliados de forma independente por esses profissionais, tendo sido feita a validação dos instrumentos intra e interavaliadores em estudo piloto anteriormente à sua aplicação. Foram excluídos da amostra os indivíduos com diagnóstico e histórico de deficiências adquiridas causadas por trauma ou doenças crônicas e/ou associadas ao envelhecimento (acidentes de trânsito ou por violência; quedas ou ferimentos; acidente vascular encefálico; entre outros) e aqueles que declinaram ao convite de participar da pesquisa e/ou não puderam participar das ações de avaliação pelos especialistas.

\section{Protocolo de avaliação físico/funcional}

Este protocolo foi desenvolvido para coleta de informações socioeconômicas; características da deficiência, etiologia e diagnóstico; nível de comprometimento motor e funcional e as condições e acesso aos serviços de reabilitação e tecnologia assistiva. A ficha tinha um cabeçalho no qual são inseridas informações para identifica- ção dos pacientes; determinação de idade em anos, gênero, escolaridade mensurada em anos de estudo; a renda familiar medida em número de salários mínimos e o número de pessoas que vivem com essa renda (renda por pessoa em reais); se o paciente tem uma atividade remunerada ou recebia benefício ou aposentadoria do Estado; se é solteiro, viúvo ou desquitado; ou mantém uma união estável. Na ficha, também foi anotado o diagnóstico clínico-genético determinado pelos médicos especialistas. A renda dos pacientes foi mensurada em número de salários mínimos, cujo valor unitário considerado foi de $\mathrm{R} \$ 622,00$ (seiscentos e vinte e dois reais) ou aproximadamente US\$ 354 (trezentos e cinquenta e quatro dólares americanos).

Posteriormente ao preenchimento desses dados socioeconômicos, os pacientes eram avaliados ou inquiridos pela equipe de fisioterapia e terapia ocupacional para determinação dos seguintes parâmetros:

- Tipo da Deficiência: se o paciente apresentava somente uma deficiência motora ou ela estava associada à perda auditiva, visual ou intelectual.

- Topografia: localização do déficit de força, se ela acometeu um lado do corpo (hemiparesia); em dois membros (diparesia); os quatro membros (tetraparesia) ou apenas um membro (monoparesia).

- Perda de função: se a função dos membros foi perdida de forma súbita, ou progressiva ou ao nascimento (congênita); e a idade em que foram observados os primeiros sintomas.

. Membros Inferiores e Superiores: foram avaliadas a presença ou ausência dos membros; ou alternativamente o nível de comprometimento de 14 parâmetros funcionais: se havia boa função dos membros ("S" para sim, os membros têm boa função; "N" para não e "P" quando a função é precária); incoordenação; movimentos involuntários; distonia; flacidez-hipotonia; nível de espasticidade (leve, moderado ou grave); rigidez articular; deformidades ósseas que eram descritas; deformidades articulares (leve, moderada ou grave); déficit de força muscular; encurtamentos; atrofia muscular, hiperreflexia ou hiporreflexia; controle cervical e de tronco ( $\operatorname{sim}$, não ou precário); deformidades da coluna (cifose, escoliose ou lordose) e se o paciente possuía histórico de episódios de queda.

- Outros Distúrbios: alterações associadas à lesão ou quadro clínico (epilepsia; presença de úlcera de pressão; disfagia; disartria; malformação craniana; hidrocefalia; nistagmo; diplopia; perda de peso; câimbras; tremores e bradicinesia). 
. Etiologia: se a lesão foi decorrente de trauma ou infecção, ocorridas durante o período pré natal; peri ou pós natal; ou se foi um trauma após o nascimento causado por acidente de trânsito, violência ou ocupação; se foi algum agente infeccioso ou sabidamente uma doença genética.

. Reabilitação: identificado se o paciente fazia, no momento da entrevista, algum tratamento médico, de reabilitação como fisioterapia e/ou terapia ocupacional; sendo anotado o número de sessões por semana, quando realizava. Se o atendimento era público ou privado e se era próximo da residência do paciente. Além disso, era sugerida prescrição de fisioterapia para os pacientes, especificando o número de sessões semanais necessárias. Foi investigado se o paciente era transportado em veículo próprio, em ambulância, carro da prefeitura, ou taxi incluindo motocicletas que fazem esse serviço nas cidades; e também foi estimado o custo desse transporte.

- Uso de Tecnologia Assistiva: foi perguntado ao paciente se ele possuía cadeira de rodas ou outros equipamentos de tecnologia assistiva (muleta axilar, muleta canadense, bengala, andador, carrinho ou skate, órteses para membros superiores e inferiores e goteira); e avaliado se ele tinha a necessidade de utilizar algum desses equipamentos para estimar custos de sua aquisição pelo Estado. Foi averiguado, no caso dos pacientes possuírem os equipamentos, se a aquisição foi feita com recursos pessoais ou por meio de doação ou concessão por terceiros ou pelo Estado.

. Rotina do paciente: investigou-se se os pacientes estudavam, trabalhavam ou tinham alguma atividade diária; se a calçada, sua residência, escola e outros ambientes eram acessíveis e permitiam, por exemplo, o tráfego de cadeira de rodas. Se eles tinham atividades de lazer passivas (assistir televisão ou ler, por exemplo) ou ativas que implicam em deslocamento do paciente. Foi registrado se os pacientes possuem amigos e quem são esses amigos (familiares, vizinhos, conhecidos da escola) e quantas vezes esses amigos costumam visitar os pacientes; e também se eles participam de atividades sociais como festas, cultos em igrejas; atividades comunitárias ou na casa de seus amigos.

Todos os pacientes foram avaliados pela fisioterapeuta e terapeuta ocupacional para determinar se precisavam de tratamento de fisioterapia ou para prescrição de equipamentos. A estimativa de custos para oferta desses serviços foi feita a partir de condições padronizadas: supondo o número médio de duas sessões semanais por paciente; que cada profissional realizasse 40 aten- dimentos por semana em regime de trabalho de 30 horas de trabalho semanal; e que fossem realizadas oito sessões de 40 minutos por dia. Considerando esses parâmetros, foram calculados quantos profissionais seriam necessários para sanar a demanda de reabilitação. O custo de transporte dos pacientes para realizar tratamentos foi estimado a partir da média dos valores relatados. Foi criado um parâmetro para cálculo de deslocamento no município de origem e para outros que disponibilizam o serviço.

A estimativa de custos para aquisição de equipamentos de tecnologia assistiva foi feita considerando a média de valores disponíveis no mercado, convertida em dólar para futuras comparações. Uma cadeira de rodas adaptada custa em média aproximadamente $\mathrm{R} \$ 2.500,00$; muletas, andadores, cadeira de banho, prancha de comunicação e carrinho de bebê foram estimados em $\mathrm{R} \$ 150,00$; parapodium, carteira escolar e órteses cerca de R \$300,00. Para conversão em dólar, foi usado o valor de $\mathrm{R} \$ 1,76$ para US $\$ 1,00$.

Os dados foram tabelados e a análise estatística descritiva feita com uso do software SPSS versão 17. O projeto foi iniciado após aprovação pelo Comitê de Ética em Pesquisa com Seres Humanos da Universidade Estadual da Paraíba, com devida autorização das instituições e gestores envolvidos; bem como leitura e assinatura do termo de consentimento livre e esclarecido pelos pacientes e seus familiares.

\section{Resultados}

Os 123 pacientes avaliados neste trabalho foram subdivididos em três grupos de afecções segundo etiologia (Doenças Genéticas, Adquiridas e Outras Afecções) e oito subgrupos das doenças prevalentes diagnosticadas na população amostrada:

a) Doenças Genéticas: Atrofia Espinal Progressiva (AEP) (6 afetados); Ataxias Espinocerebelares, reunindo síndrome de Machado-Joseph ou SCA3 $(\mathrm{N}=23)$ e Ataxia Telangiectasia $(\mathrm{N}=$ 02); Distrofia Muscular do tipo Becker $(\mathrm{N}=3)$, Cinturas do tipo II-B (disferlinopatias) $(\mathrm{N}=10)$ e Steinert ( $\mathrm{N}=3)$; Spoan (3); Outras Síndromes Genéticas $(\mathrm{N}=15)$.

b) Doenças Adquiridas: Encefalopatia não progressiva $(\mathrm{ENP})(\mathrm{N}=37)$; Sequela de Poliomielite (Pólio) $(\mathrm{N}=6)$

c) Outras afecções: grupo no qual foram reunidos os quadros de malformação ou e demais afecções $(\mathrm{N}=17)$. 
Na Tabela 1 está descrita a caracterização socioeconômica dessa população. Verificou-se que a proporção de homens na amostra está acima do esperado $(60,2 \%)$. Isto pode ser explicado pelo fato de que algumas doenças possuem padrão de herança ligada ao $\mathrm{X}$, como a distrofia muscular do tipo Becker, afetando mais frequentemente homens. No grupo das ataxias e AEP, foram encontradas quase o dobro de pessoas do gênero masculino afetadas sem que isto tenha causa específica determinada.

Em relação à faixa etária, não foram encontrados pacientes afetados por encefalopatia crô- nica não progressiva com mais de 45 anos, embora eles constituam $30 \%$ da amostra. Somente $2,4 \%$ da população têm mais de 65 anos. Também não foram identificadas pessoas com menos de 38 anos acometidos com sequela de poliomielite, pois essa doença foi erradicada há cerca de 40 anos na região estudada.

As doenças genéticas progressivas, e cujos sintomas surgem tardiamente, não impedem os afetados de constituírem união estável. Entre as pessoas com deficiências que impedem ou comprometem a marcha, cerca da metade daqueles com distrofia e ataxia são casados. Entre os afetados

Tabela 1. Características socioeconômicas da população amostrada por grupos de afecções encontrados (SM - Salários Mínimos; N - número de indivíduos). AEP: Atrofia Espinal Progressiva; ATAXIA: ataxias espinocerebelares; DISTROFIA: distrofias musculares; SPOAN: síndrome Spoan; SG: outras síndromes genéticas; ENP: Encefalopatia não progressiva; POLIO: poliomielite; OUTRAS: outros grupos de afecções.

\begin{tabular}{|c|c|c|c|c|c|c|c|c|c|c|}
\hline & $\begin{array}{c}\text { AEP } \\
\mathrm{N}\end{array}$ & $\begin{array}{c}\text { ATAXIA } \\
\mathrm{N}\end{array}$ & $\begin{array}{c}\text { DISTROFIA } \\
\mathrm{N}\end{array}$ & $\begin{array}{c}\text { SPOAN } \\
\mathrm{N}\end{array}$ & $\begin{array}{l}\text { SG } \\
\mathrm{N}\end{array}$ & $\begin{array}{c}\text { ENP } \\
\mathrm{N}\end{array}$ & $\begin{array}{c}\text { POLIO } \\
\mathrm{N}\end{array}$ & $\begin{array}{c}\text { OUTROS } \\
\mathrm{N}\end{array}$ & $\begin{array}{c}\text { Total } \\
\mathrm{N}\end{array}$ & $\%$ \\
\hline \multicolumn{11}{|l|}{ Gênero } \\
\hline Feminino & 0 & 8 & 6 & 1 & 6 & 15 & 3 & 10 & 49 & 39,8 \\
\hline Masculino & 6 & 15 & 10 & 2 & 9 & 22 & 3 & 7 & 74 & 60,2 \\
\hline Total & 6 & 23 & 16 & 3 & 15 & 37 & 6 & 17 & 123 & 100 \\
\hline \multicolumn{11}{|l|}{ Faixa etária } \\
\hline ID 0 a 5 & 0 & 0 & 1 & 0 & 2 & 5 & 0 & 0 & 8 & 6,5 \\
\hline ID 6 a 15 & 0 & 2 & 1 & 1 & 6 & 16 & 0 & 0 & 26 & 21,1 \\
\hline ID 16 a 45 & 4 & 13 & 10 & 2 & 7 & 16 & 4 & 13 & 69 & 56,1 \\
\hline ID 46 a 65 & 2 & 7 & 3 & 0 & 0 & 0 & 2 & 3 & 17 & 13,8 \\
\hline ID $>65$ & 0 & 1 & 1 & 0 & 0 & 0 & 0 & 1 & 3 & 2,4 \\
\hline Total & 6 & 23 & 16 & 3 & 15 & 37 & 6 & 17 & 123 & 100 \\
\hline \multicolumn{11}{|l|}{ Estado civil } \\
\hline Casado* & 3 & 12 & 7 & 0 & 0 & 0 & 3 & 4 & 30 & 24,4 \\
\hline Solteiro* & 3 & 11 & 9 & 3 & 15 & 37 & 3 & 13 & 92 & 74,8 \\
\hline Total & 6 & 23 & 16 & 3 & 15 & 37 & 6 & 17 & 123 & 100 \\
\hline \multicolumn{11}{|l|}{ Escolaridade } \\
\hline Nenhuma & 3 & 3 & 1 & 1 & 11 & 32 & 2 & 7 & 60 & 52,6 \\
\hline 01 a 04 anos & 1 & 10 & 2 & 1 & 1 & 3 & 1 & 0 & 19 & 16,7 \\
\hline 05 a 08 anos & 0 & 5 & 8 & 0 & 1 & 2 & 2 & 4 & 22 & 19,3 \\
\hline 09 a 12 anos & 2 & 5 & 2 & 0 & 1 & 0 & 1 & 2 & 13 & 11,4 \\
\hline Total & 6 & 23 & 13 & 2 & 14 & 37 & 6 & 13 & $114^{* *}$ & 100 \\
\hline \multicolumn{11}{|l|}{ Benefício } \\
\hline Não tem & 0 & 4 & 3 & 3 & 4 & 1 & 0 & 4 & 19 & 16,5 \\
\hline Tem & 6 & 19 & 12 & 0 & 8 & 34 & 5 & 12 & 96 & 83,5 \\
\hline Total & 6 & 23 & 15 & 3 & 12 & 35 & 5 & 16 & $115^{\star *}$ & 100 \\
\hline \multicolumn{11}{|l|}{ Renda } \\
\hline Nenhum & 0 & 0 & 0 & 0 & 1 & 0 & 0 & 0 & 1 & 0,9 \\
\hline $1 \mathrm{SM}$ & 5 & 9 & 7 & 3 & 5 & 21 & 3 & 8 & 61 & 56,0 \\
\hline $2 \mathrm{SM}$ & 0 & 4 & 3 & 0 & 0 & 10 & 1 & 2 & 20 & 18,3 \\
\hline $3 \mathrm{SM}$ & 1 & 2 & 2 & 0 & 4 & 4 & 1 & 3 & 17 & 15,6 \\
\hline $4 \mathrm{SM}$ & 0 & 6 & 1 & 0 & 0 & 0 & 0 & 3 & 10 & 9,2 \\
\hline Total & 6 & 21 & 13 & 3 & 10 & 35 & 5 & 16 & $109^{* *}$ & 100 \\
\hline
\end{tabular}

* casado ou mantém união estável; solteiro, viúvo ou desquitado. ${ }^{* *}$ alguns participantes não souberam e/ou permitiram acesso à informação. 
com paralisia cerebral (PC) não foram encontrados indivíduos casados.

Metade da população amostrada não tem nenhuma escolaridade, somente $11,4 \%$ alcançaram o ensino médio e nenhum deles realizou um curso em nível de ensino superior. Verificou-se que $83,5 \%$ recebem benefício ou aposentadoria do Estado e $56 \%$ da população relataram que a família vive com apenas um salário mínimo. A renda média aferida a partir dos relatos dos pacientes foi de 1,8 salários por domicílio para subsidiar os custos de 3,8 pessoas em média. O máximo de renda referida por $9,2 \%$ da amostra foi de quatro salários.

\section{Caracterização das deficiências}

A aplicação do protocolo de avaliação físico/ funcional permitiu caracterizar melhor as perdas e os níveis de comprometimento motor da população amostrada, como mostrado na Tabela 2. É possível observar que $68 \%$ dos indivíduos apresentam apenas deficiência motora enquanto $28,5 \%$ apresentam perda motora associada à intelectual. Interessantemente, $48,8 \%$ das doenças são progressivas e $58,5 \%$ delas são de- terminadas por fatores genéticos. Isto significa que, para as populações com elevadas frequências de casamentos consanguíneos, as doenças genéticas podem ser mais frequentes do que, por exemplo, as encefalopatias não progressivas $(30 \%)$ dentre as quais o quadro mais comum encontrado foi de paralisia cerebral.

$\mathrm{Na}$ população avaliada, $74 \%$ dos indivíduos eram tetraparéticos; $42,3 \%$ não tinha função de membros inferiores e dependiam de cadeira de rodas para locomoção; enquanto $25,2 \%$ não tinha função de membros superiores e, portanto, essas pessoas dependiam do cuidado de terceiros. Somente $37,4 \%$ da população amostrada não possuía nenhuma deformidade de coluna vertebral. Aproximadamente $30 \%$ da amostra apresenta algum distúrbio associado, como disfagia, disartria e/ou problemas respiratórios. Em algumas doenças, como as ataxias espinocerebelares (SCA3), foi identificada a necessidade de gastrostomia.

\section{Demandas por serviços especializados}

Dos 123 pacientes avaliados, apenas 23 realizavam sessões de fisioterapia no momento desta investigação, correspondendo a 19\% da popula-

Tabela 2. Caracterização das perdas que causam deficiência motora com uso do protocolo de avaliação físico/funcional.

\begin{tabular}{|c|c|c|c|c|c|}
\hline & $\mathbf{N}$ & $\%$ & & $\mathbf{N}$ & $\%$ \\
\hline 1. Tipo de Deficiência & & & 6. Controle Cervical & & \\
\hline Somente motora & 123 & 100,0 & Tem boa função & 88 & 71,5 \\
\hline Motora e Intelectual & 35 & 28,5 & Não tem função & 15 & 12,2 \\
\hline Motora e Visual & 3 & 2,4 & A função é precária & 20 & 16,3 \\
\hline Motora e Auditiva & 1 & 0,8 & 7. Controle de Tronco & & \\
\hline 2. Topografia & & & Tem boa função & 71 & 57,7 \\
\hline Tetraparesia & 91 & 74,0 & Não tem função & 23 & 18,7 \\
\hline Diparesia & 25 & 20,3 & A função é precária & 29 & 23,6 \\
\hline Hemiparesia & 4 & 3,3 & 8. Coluna & & \\
\hline Monoparesia & 3 & 2,4 & Cifose & 29 & 23,6 \\
\hline 3. Perda de função & & & Escoliose & 45 & 36,6 \\
\hline Progressiva & 60 & 48,8 & Lordose & 3 & 2,4 \\
\hline Ao nascimento & 52 & 42,3 & 9. Distúrbios Associados & & \\
\hline Súbita & 11 & 8,9 & Respiratórios & 39 & 31,7 \\
\hline 4. Função Membros Superiores & & & Disartria & 47 & 38,2 \\
\hline Tem boa função & 56 & 45,5 & Disfagia & 41 & 33,3 \\
\hline Não tem função & 31 & 25,2 & Convulsão & 14 & 11,4 \\
\hline A função é precária & 36 & 29,2 & Nistagmo & 14 & 11,4 \\
\hline 5. Função Membros Inferiores & & & Outros & 14 & 11,4 \\
\hline Tem boa função & 29 & 23,6 & 10. Etiologia & & \\
\hline Não tem função & 52 & 42,3 & Pré, peri e pós natal & 35 & 28,5 \\
\hline \multirow[t]{2}{*}{ A função é precária } & 42 & 34,1 & Infecciosa & 11 & 8,9 \\
\hline & & & Genética & 72 & 58,5 \\
\hline
\end{tabular}


ção amostrada; e nenhum deles relatou a realização de outros tratamentos como terapia ocupacional ou fonoaudiologia. Desse total, 68 tinham indicação para realizar em média duas sessões de fisioterapia por semana, portanto 91 pacientes deveriam fazer atendimento fisioterápico.

Para atender essa demanda, foi estimado que seriam necessários de 4 a 5 fisioterapeutas para atender 20 pessoas por semana em centros de referência, havendo necessidade de deslocamento dos pacientes. No caso da população amostrada, tendo em vista que eles têm total dependência ou dependência moderada, para que os pacientes realizem algum atendimento, eles devem ser acompanhados pelo cuidador e ter acesso ao transporte público e/ou oferecido pela prefeitura.

O custo de transporte médio estimado foi de $\mathrm{R} \$ 10,00$ por viagem para deslocamento no próprio município e $\mathrm{R} \$ 50,00$ para atendimento em outro município vizinho. A maioria dos pacientes vive em regiões rurais de difícil acesso e somente $28 \%$ residem em áreas com ruas pavimentadas. Essas condições dificultam o acesso dos pacientes aos serviços, especialmente quando são ofertados fora do seu município de origem; o que explicaria o fato de muitos deles terem relatado o abandono do tratamento. Considerando as condições de acesso e seu quadro clínico, a melhor alternativa para os pacientes seria o atendimento de reabilitação domiciliar. Neste caso, a estimativa de número de fisioterapeutas para atender a essa demanda se elevaria.

Em relação aos equipamentos, 46 pacientes utilizavam cadeira de rodas e somente duas delas foram compradas com recursos próprios; enquanto 28 indivíduos precisavam desse equipamento e não tinham condições financeiras para efetivar a compra. Ao todo, foram prescritas 67 cadeiras de rodas adaptadas e um triciclo, cujo custo para compra foi estimado em R $\$ 170.000,00$. Considerando as demandas por outros equipamentos, como andadores (10), prancha de comunicação (5), cadeira de banho (4) e órteses (4), muletas (3), bengalas (2), carteira escolar (1), parapodium (1), muleta (1), e carrinho de bebe (1); então o custo dos equipamentos aumentaria em $\mathrm{R} \$ 4.950,00$. Considerando que a renda média dos pacientes foi de 1,8 salários mínimos mensais, dificilmente eles conseguirão adquirir esses equipamentos; daí a necessidade de estruturação do serviço de órtese do Estado para atender a essa demanda.

\section{Discussão}

A endogamia é um fator de risco para manifestação de doenças genéticas que causam diferentes deficiências ${ }^{14-17}$. Estudos epidemiológicos nos municípios do sertão do Rio Grande do Norte revelaram algumas doenças genéticas prevalentes naquelas populações, como a síndrome Spoan ${ }^{12,13}$ e que as deficiências motoras representam de 9,5\% a 28,4\% da totalidade das deficiências que afetam aquelas populações ${ }^{10}$. Entretanto, até o presente momento, pouco se sabe a respeito da contribuição de diferentes fatores etiológicos para determinação das diferentes formas de deficiência.

Nesta pesquisa, realizado em oito municípios do estado da Paraíba limítrofes com a região onde a síndrome Spoan é prevalente, foram descobertas três pessoas afetadas com essa síndrome indicando que a área de abrangência dessa doença é ainda maior do que previamente relatado.

Além da síndrome Spoan, foram descobertas três outras doenças genéticas prevalentes nessa população, duas delas de herança recessiva cuja manifestação está associada à endogamia: a amiotrofia espinal progressiva causada pela deleção dos exons 7 e 8 do gene SMN1 e distrofia muscular do tipo cinturas II-B (disferlinopatia). A ataxia espinocerebelar tipo 3 ou síndrome de Machado-Joseph ${ }^{18}$, cuja mutação fundadora encontra-se ainda em investigação, possui padrão de herança dominante; portanto, metade da prole dos afetados carrega a mutação que causa a doença. É provável que novos casos sejam relatados ao longo das próximas gerações ou talvez a área de abrangência seja maior do que a região investigada neste trabalho.

As doenças genéticas são responsáveis por praticamente $60 \%$ das deficiências que causam perda da função motora da amostra selecionada para estudo e outros 30\% são decorrentes possivelmente de intercorrências durante a gestação e/ ou período peri e pós-natal. Nos países subdesenvolvidos, como no Brasil, a paralisia cerebral pode estar relacionada a problemas gestacionais, desnutrição materna e infantil, e atendimento médico e hospitalar inadequado ${ }^{19}$. Diferentemente do que seria esperado, essas doenças adquiridas não foram o principal fator etiológico encontrado entre as pessoas com deficiência motora incapacitante.

Sabe-se que a Paraíba possui a maior taxa de pessoas com deficiência do país ${ }^{2}$, menor renda média da população e também a menor escolaridade - apenas 2,8 anos de escolarização em média. A análise da relação entre deficiência e renda 
familiar deve ser interpretada como uma associação e não necessariamente uma causa ou consequência ${ }^{20}$. Se considerarmos que a pobreza apresenta também assimetrias no próprio estado da Paraíba, as populações que vivem no sertão deveriam ter risco maior de manifestar ou adquirir alguma deficiência.

Mais pessoas com deficiência são pobres e por esta situação têm mais risco de serem acometidas por alguma deficiência. Má nutrição, padrões de vida, acesso precário aos serviços de saúde e atendimento à gestante estão entre os fatores que contribuem para isso. O baixo peso ao nascer, vacinação, altas taxas de analfabetismo, vulnerabilidade dos empregos também elevam as taxas de deficiência ${ }^{21}$. Essa relação é descrita como "círculo vicioso" que tornam as pessoas com deficiência entre os mais pobres dos pobres. Uma família com uma pessoa acometida por uma deficiência geralmente têm mais custos e menor renda per capita.

A população amostrada neste estudo possui deficiências que comprometem severamente a realização das atividades da vida diária. Além disso, foi estimado que a renda média das famílias é de 1,8 salários mínimos para custear 3,8 pessoas. Sabe-se que existe um "círculo vicioso" entre pobreza, educação e deficiência: as famílias com pessoas deficientes perdem duplamente porque os deficientes não podem desenvolver atividades remuneradas e produtivas, dependendo de familiares para seu cuidado, o que reduz ainda mais a renda da família. São, ao menos, duas pessoas que não trabalham para gerar renda.

Por outro lado, as famílias pobres que não têm acesso aos serviços de saúde de qualidade e vivem em situações de violência têm maior chance de apresentar deficiências adquiridas. Vimos, por exemplo, que $30 \%$ das deficiências são adquiridas durante o período pré, peri ou pós-natal; e possivelmente uma parte delas poderia ser evitada com oferta de serviços de saúde adequados na assistência materno-infantil.

Por outro lado, nos casos das deficiências causadas por doenças genéticas, é comum que em uma irmandade tenha mais de um indivíduo afetado. Essa condição de pobreza da população com deficiência restringe seu acesso à reabilitação existente nos centros mais distantes das capitais. A falta de assistência de uma equipe multiprofissional especializada inviabiliza intervenções preventivas e aquisição de equipamentos de tecnologia assistiva, como a cadeira de rodas adaptadas. Também distancia as famílias de orientações e conhecimentos que poderiam subsi- diar decisões reprodutivas, como o aconselhamento genético.

Neste trabalho, foram avaliados 123 pacientes com deficiência física, que dificulta ou impede a sua locomoção, de uma população de 50 mil habitantes; e foi estimado que seriam necessários cerca de $\mathrm{R} \$ 175.000,00$ (cento e setenta e cinco mil reais) de investimentos para suprir demandas de equipamentos de tecnologia assistiva. Supondo os indivíduos que não foram avaliadas pelos especialistas representasse $15 \%$ da amostra; poderíamos partir do pressuposto que seriam necessários, no mínimo, duzentos mil reais para sanar as demandas de tecnologia assistiva das comunidades de oito municípios da Paraíba.

Caso a prevalência de deficiência física e as demandas pelos serviços de tecnologia assistiva da população do Estado da Paraíba, com aproximadamente três milhões de pessoas, fosse a mesma daquela encontrada na população amostrada, poderíamos estimar que seriam necessários 60 vezes o valor referido, ou seja, cerca de 12.000.000,00 (doze milhões de reais) para aquisição de cadeiras de rodas e outros equipamentos para as pessoas com deficiência física de todo o Estado. Se a demanda por fisioterapeutas também fosse semelhante à da amostra, então seriam necessários, no mínimo, 300 profissionais fisioterapeutas. Trata-se de uma estimativa para alguns serviços específicos que nos permitem refletir a respeito de quanto custaria ao Estado sanar algumas das necessidades das pessoas que têm muita dificuldade ou incapacidade de locomover-se.

Estratégias alternativas e políticas públicas descentralizadas precisam ser estabelecidas nos sistemas de saúde em seus diferentes níveis de organização para atender às demandas das pessoas com deficiência. A incorporação, por exemplo, de fisioterapeutas e do terapeuta ocupacional na Estratégia de Saúde da Família; o estímulo à criação de centros de referência em Genética Médica no interior dos estados do Nordeste e à formação de consórcios municipais e com o Estado para aquisição de equipamentos de tecnologia assistiva; bem como o estabelecimento de unidades de referência regionais para oferecer reabilitação no interior dos estados com equipe interdisciplinar com médicos, fisioterapeutas, terapeutas ocupacionais, fonoaudiólogos, psicólogos, assistentes sociais, educadores e demais profissionais da saúde são necessários para melhorar a condição de vida das pessoas com deficiência que vivem em regiões rurais e pobres, especialmente no nordeste brasileiro. 
e recomendaçóes para estabelecimento políc públicas que facilitem o acesso das populações em risco para manifestação de doenças genéticas aos serviços de Genética Médica, como aquelas descritas neste trabalho. Nesse documento, intitulado "Community genetics services: Report of a WHO Consultation on community genetics in low- and middle-income countries", vários especialistas recomendam fortemente o investimento em estudos epidemiológicos para determinação da prevalência de doenças genéticas e demandas por serviços específicos. No Brasil, embora tenha sido regulamentada a Política Nacional de Atenção Integral em Genética Clínica no SUS por meio da portaria no 81 de 20 de janeiro de 2009; os poucos serviços de Genética Clínica - e não Genética Comunitária na perspectiva da $\mathrm{WHO}$ estão concentrados nos estados do sudeste e são oferecidos principalmente por pesquisadores em centros de referência universitários ${ }^{22-26}$.

A maioria dos pacientes participantes desta pesquisa não teve, por um lado, acesso a um ser-

\section{Colaboradores}

S Santos trabalhou na concepção, coleta e consolidação de dados e redação do artigo; AAS Pequeno e CRC Galvão elaboraram o protocolo, realizaram a avaliação funcional, a consolidação, análise dos dados, e redação do artigo; ALS Pessoa e JLA Medeiros realizaram avaliação neurológica; ES Almeida e JC Pereira participaram das atividades de coleta e consolidação de dados; F Kok foi responsável pela avaliação clínica e revisão do artigo. viço médico especializado para diagnóstico e tratamento adequado; também não tiveram acesso ao aconselhamento genético que é importante tendo em vista os altos riscos de recorrência das doenças genéticas nas famílias nas quais segregam as mutações. Por outro lado, as políticas públicas existentes no Estado da Paraíba para oferta de equipamentos de tecnologia assistiva, como cadeiras de rodas adaptadas, ainda não conseguem responder às demandas existentes.

Por força da Portaria no 1097 de maio de 2006, que define a Programação Pactuada e Integrada (PPI), a alocação de recursos para aquisição de Órteses, Próteses e Materiais Especiais (OPM) ficou sob a responsabilidade dos municípios, que recebem um montante geralmente inferior ao necessário para responder à demanda. Quando esses municípios conseguem responder a uma solicitação, como o SUS financia apenas a aquisição de cadeiras de rodas ditas padrão, as pessoas com deficiência recebem equipamentos que necessitam de adaptações para cumprir sua função plenamente. Tais serviços especializados têm custo elevado e, como vimos, não podem ser financiados pela população que dele tanto necessita e nem pelos municípios, sobre os quais recai tal responsabilidade. As políticas existentes, portanto, não respondem adequadamente às necessidades das pessoas com deficiência.

\section{Agradecimentos}

Ao Prof. Dr. Paulo Alberto Otto por sua disposição em realizar avaliação genética dos pacientes deste estudo e a todas as prefeituras e profissionais da saúde que participaram deste estudo. Especialmente à Valéria Ravani Soares da Biomarin do Brasil pelo apoio financeiro. 


\section{Referências}

1. Instituto Brasileiro de Geografia e Estatística (IBGE). Censo Demográfico 2000. Rio de Janeiro: IBGE; 2001.

2. Néri M. Retratos da Deficiência no Brasil. Rio de Janeiro: FGV, IBRE, CPS; 2003.

3. Instituto Brasileiro de Geografia e Estatística (IBGE). Censo Demográfico 2010: Resultados Preliminares. [página na Internet]. [acessado $2011 \mathrm{dez} 15]$. Disponível em: http://www.ibge.gov.br/home/estatistica/ populacao/censo2010/default.shtm

4. Trani JF, Loeb M. Poverty and Disability: A Vicious Circle? Evidence from Afghanistan and Zambia. J. Int. Dev. 2010; 24(Supl. 1):S19-S52.

5. Freire-Maia N. Inbreeding in Brazil. Am. J. Hum. Gen. 1957; 9(4):284-98.

6. Freire-Maia N. Genetic Effects in Brazilian Populations due to Consanguineous Marriages. Am J Med Gen 1989; 35(1):115-117.

7. Fonseca LG, Freire-Maia N. Further Data on Inbreeding Levels in Brazilian Populations. Soc Biol 1970; 17(4):324-328.

8. Orioli IM, Castilla EE, Carvalho WP. Inbreeding in a South American Newborn Series. Acta Anthropogenetica 1982; 6(1):45-55.

9. Santos S, Kok F, Weller M, Paiva FRL, Otto, PA Inbreeding Levels in Northeastern Brazil: Strategies for the Prospection of New Genetic Disorders. Genetics and Molecular Biology 2010; 33(2):1-2.

10. Santos S, Melo US, Lopes SSS, Weller M, Kok F. A Endogamia Explicaria a Elevada Prevalência de Deficiências em Populações do Nordeste Brasileiro? Cien Saude Colet 2012; 18(4):1141-1150.

11. Weller M, Tanieri M, Pereira JC, Almeida ES, Kok SS, Santos S. Consanguineous Unions and the Burden of Disability: A Population-based Study in Communities of Northeastern Brazil. Am J Hum Biol 2012; 24(6):835-840.

12. Macedo-Souza LI, Kok F, Santos S, Amorim S, Starling A, Nishimura A, Lezirovitz K, Lino AMM, Zatz M. Spastic Paraplegia, Optic Atrophy, and Neuropathy is Linked to Chromosome 11q13. Ann Neurol 2005; 57(5):730-737.

13. Macedo-Souza LI, Kok F, Santos S, Licinio L, Lezirovitz K, Cavaçana N, Bueno C, Amorim S, Pessoa A Graciani Z, Ferreira A, Prazeres A, Melo AN, Otto PA, Zatz M. Spastic Paraplegia, Optic Atrophy, and Neuropathy: New Observations, Locus Refinement, and Exclusion of Candidate Genes. Ann Hum Genet 2009; 73(Pt 3):382-387.

14. Bittles AH. Consanguineous Marriage: Current Global Incidence and its Relevance to Demographic Research. PSC Research Report No. 90-186. August 1990.
15. Bittles AH. The role and significance of consanguinity as a demographic variable. Popul. Develop. Review 1994; 20(3):561-584.

16. Liascovich R, Rittler M, Castilla EE. Consanguinity in South America: Demographic Aspects. Hum. Heredit. 2001; 51(1-2):27-34.

17. Al-Kandari Y, Crews DE. The Effects of Consanguinity on Congenital Disabilities in the Kuwaiti Population. J. Bios. Sci. 2011; 43(1):65-73.

18. Bettencourt C, Lima M. Machado-Joseph Disease: From First Descriptions to new Perspectives. Orphanet J Rare Dis 2011; 6:35.

19. Mancini MC, Alves ACM, Schaper C, Figueiredo EM, Sampaio RF, Coelho ZAC, Tirado MGA. Gravidade da Paralisia Cerebral e Desempenho Funcional. Rev. Bras. fisioter. 2004; 8(3):253-260.

20. Filmer D. The World Bank. Disability, Poverty and Schooling in Developing Countries: Results from 11 Household Surveys. Washington: The World Bank; 2005. [SP Discussion Paper]

21. Palmer, M. Disability and Poverty: A Conceptual Review. Journal of Disability Policy Studies 2011; 21(4):210-218.

22. World Health Organization (WHO). Community Genetics Services: Report of a WHO Consultation on Community Genetics in Low-and Middle-income Countries, Geneva: WHO; 2010.

23. Beiguelman B. Human and Medical Genetics in Brazil. Genetics and Molecular Biology 2000; 23(2): 277-281.

24. Marques-de-Faria AP, Ferraz VEF, Acosta AX, Brunoni D. Clinical Genetics in Developing Countries: The Case of Brazil. J Community Genet 2004; 7(2-3):95-105.

25. Horovitz DDG, Junior JCL, Mattos MA. Birth Defects and Health Strategies in Brazil: an Overview. Cad Saude Publica 2006; 21(4):2599-2609.

26. Novoa MC, Burnham TF. Challenges for the Universalization of Clinical Genetics: The Brazilian Case. Rev Panam Salud Publica 2011; 29(1):61-68.

Artigo apresentado em 18/01/2013

Aprovado em 11/03/2013

Versão final apresentada em 15/03/2013 